\title{
Controle da disfunção temporomandibular utilizando a laserterapia
}

\author{
Control of temporomandibular dysfunction using laserterapy
}

Control de la disfunción temporomandibular mediante laserterapia

\author{
Matheus Cavalcante Rodrigues de Lima1*, Islaine Sant'Anna Valoz', Katharina Jucá de Moraes \\ Fernandes ${ }^{1}$, Fernanda Braga Peixoto ${ }^{1}$.
}

\section{RESUMO}

Objetivo: Realizar uma análise na literatura científica a respeito do tratamento da Disfunção Temporomandibular (DTM), utilizando a técnica de Laserterapia de Baixa Intensidade (LBI), executado pelo Cirurgião-Dentista (CD). Métodos: Como forma de pesquisa foram determinadas pesquisas nas bases de dados PubMed/MedLine, LiLacs e SciELo, no período de 2016-2021, combinando os descritores "Terapia a laser", "Terapia com luz de baixa intensidade", "Transtornos da Articulação Temporomandibular" nas específicas fontes de pesquisa. Resultados: A utilização da LBI vem sendo administrada para o tratamento da DTM, tendo como um dos principais critérios o alívio das dores agudas ou crônicas que os pacientes apresentam, sendo observado que depois de algumas sessões ocorre a diminuição de processos inflamatórios presentes no local estabelecido e a restauração da função. Esse tratamento terapêutico tem ofertado alto grau de segurança tanto para o paciente, quanto para o profissional, visto que não apresenta conduta invasiva, muito menos contraindicações, sendo também de fácil manuseio pelo profissional qualificado. Considerações finais: $A$ aplicação da LBI é realizada com frequência pelo CD, apresentando resultados satisfatórios no tratamento da DTM por não possuir efeitos colaterais, tornando-a confiável. No entanto, os profissionais devem estar aptos a realizar uma técnica adequada e satisfatória, visando o bem-estar do paciente.

Palavras-chave: Terapia a laser, Terapia com luz de baixa intensidade, Transtornos da articulação temporomandibular.

\section{ABSTRACT}

Objective: Perform an analysis in the scientific literature regarding the treatment of Temporomandibular Disorder (DTM), using the low-level laser therapy (LBI) technique performed by the Dental Surgeon (CD). Methods: As a form of research, research was determined in the PubMed / MedLine, LiLacs and SciELo databases, in the period 2016-2021, combining the descriptors "Laser therapy", "Low intensity light therapy", "Temporomandibular Joint Disorders" in specific research sources. Results: The use of LBI has been administered for the treatment of DTM, having as one of the main criteria the relief of acute or chronic pain that patients have, and it is observed that after a few sessions there is a decrease in inflammatory processes present in the established site and restoration function, this therapeutic treatment has offered a high degree of safety for both the patient and the professional, since it does not present invasive conduct, much less contraindications and easy handling by the qualified professional. Final considerations: The application of LBI is a treatment frequently performed by DC, of which they present satisfactory results in the treatment of DTM, as it does not present side effects, it becomes reliable, however professionals must be able to perform an adequate and satisfactory technique, aiming the patient's well-being.

Keywords: Laser therapy, Low-level light therapy, Temporomandibular joint disorders.

\section{RESUMEN}

Objetivo: Realizar un análisis en la literatura científica sobre el tratamiento de la Disfunción Temporomandibular (DTM), utilizando la técnica de la terapia con láser de bajo nivel (LBI) realizada por el Cirujano Dentista (CD). Métodos: Como forma de investigación, la investigación se determinó en las bases de datos PubMed / MedLine, LiLacs y SciELo, en el período 2016-2021, combinando los descriptores "Terapia láser", "Terapia de luz de baja intensidad", "Trastornos de la articulación temporomandibular" en investigaciones específicas. fuentes. Resultados: El uso de LBI se ha administrado para el tratamiento de los DTM, teniendo como uno de los principales criterios el alivio del dolor agudo o crónico que presentan los pacientes, y se observa que luego de unas pocas sesiones hay una disminución de los procesos inflamatorios presentes en el sitio establecido y restauración En términos de función, este tratamiento terapéutico ha ofrecido un alto grado de seguridad tanto para el paciente como para el profesional, ya que no presenta conducta invasiva, mucho menos contraindicaciones y es fácil de manejar por un profesional calificado. Consideraciones finales: La aplicación de LBI es un tratamiento frecuentemente realizado por DC, de los cuales presentan resultados satisfactorios en el tratamiento de los DTM, ya que no presenta efectos secundarios, se vuelve confiable, sin embargo los profesionales deben ser capaces de realizar una técnica adecuada y satisfactoria, buscando el bienestar del paciente.

Palabras clave: Terapia por láser, Terapia por luz de baja intensidad, Trastornos de la articulación temporomandibular.

${ }^{1}$ Centro Universitário CESMAC (CESMAC), Maceió - AL. `E-mail: matheuscavalcanterlima@gmail.com 


\section{INTRODUÇÃO}

Na última década, ocorreram diversos avanços científicos principalmente os determinados por intermédio de metodologias físicas, tendo como um dos principais objetivos contribuir com o melhoramento da tecnologia, em especial os que são procedentes dos âmbitos de diagnósticos e procedimentos terapêuticos. Uma das vantagens identificadas nessa área é estabelecida por meio da laserterapia, sendo formada por um laser do qual é um acrossílabo da língua inglesa (light amplification by stimulated emission of radiation, cuja tradução remete à ampliação de um feixe de luz por difusão determinada por radiação), sendo essa luz bem desenvolvida e precisa, em que retém inúmeros benefícios em todos os campos de estudo e foi incluída na área da saúde como um instrumento bastante satisfatório, em especial nos processos que envolvem os tratamentos realizados pelo Cirurgião-Dentista (CD) (POL R, et al., 2016; KHAW CM, et al., 2018).

A luz presente no laser exibe feixes de radiações vistos nos espectros eletromagnéticos que são diferenciados pela luminosidade ultravioleta ao infravermelho, sendo conhecida pelo espectro de feixes visíveis. As radiações ocorridas com esse feixe de luz, em processos terapêuticos, não prejudicam os pacientes, já que não apresentam características invasivas, tendo uma boa aceitabilidade (POL R, et al., 2016).

No mercado são encontrados dois tipos de laser, dos quais são utilizados no campo da saúde. Os que apresentam maiores luminosidades são utilizados na parte cirúrgica de forma conservadora, tendo um dos seus principais objetivos à diminuição das dores nos procedimentos pós-cirúrgicos. O outro tipo de laser que apresenta pequena intensidade, Low Level Laser Therapy (LLLT), é utilizado em tratamentos terapêuticos como forma de analgesia, atua na melhora da cicatrização e é um bioestimulador, apresentando efeito antiinflamatório e, além disso, detém peculiaridades benéficas em processos terapêuticos que utilizam bases fotodinâmicas, promovendo melhores resultados quando se quer tratar uma infecção (KHAW CM, et al., 2018).

Nos Estados Unidos, utiliza-se muito o laser de alta intensidade. Porém, no Brasil, ele não apresenta muita aplicabilidade. Um dos fatores que fazem com que o lazer de baixa intensidade seja mais utilizado é o fato do seu custo ser mais reduzido, tornando-o mais acessível (CIEPLIK F, et al., 2018). Na área odontológica, o laser apresenta inúmeros benefícios, dispondo de resultados positivos em cada especialização, principalmente quando se trata de problemas relacionados à Articulação Temporomandibular (ATM). Pacientes que sofrem com Disfunção Temporomandibular (DTM) apresentam segurança quanto à aplicação do laser, no entanto não é uma técnica farmacológica (SANT'ANNA EF, et al., 2017).

Os feixes de luz utilizados por esses tipos de laser atuam no melhoramento do tratamento metabólico, através da inserção de fatores energéticos feitos pelos cromóforos (conjunto de átomos de uma molécula), fazendo com que ocorra uma modificação na funcionalidade das mitocôndrias, e consequentemente, ocasionam modificações na parte respiratória das células existentes, aumentando a produtividade de Adenosina Trifosfato (ATP), determinando a intensificação de células que reagem com o oxigênio de maneira intracelular. Assim, gera a multiplicação de fibroblastos, moléculas de colágenos e, por consequência, apresenta melhora no tratamento da DTM (SANT'ANNA EF, et al., 2017).

De maneira frequente, por causa da falta de informação referente ao assunto, e pelo motivo de muitos Cirurgiões-Dentistas não saberem quais são as interações relacionadas entre o laser e problemas de DTM, como também, por consequência dos efeitos benéficos que eles apresentam em procedimentos terapêuticos, os profissionais da área acabam por não utilizarem, deixando de obter respostas mais positivas nos tratamentos feitos em seu cotidiano (SANT'ANNA EF, et al., 2017).

Nesse contexto, a premissa desse estudo apresentou como um dos principais objetivos analisar a importância da utilização do laser no tratamento de dores causadas pela DTM, através da realização de laserterapia de baixa intensidade, tendo em vista que esse processo terapêutico vem cada vez mais sendo utilizado pelos Cirurgiões-Dentistas.

\section{MÉTODOS}

A busca dessa pesquisa estabeleceu-se por meio de uma revisão integrativa efetuada entre os períodos de dezembro de 2020 até fevereiro do ano presente, em que foram explorados artigos científicos publicados 
em bases de dados eletrônicos Literatura Latino Americana e do Caribe em Ciências da Saúde (LILACS) Literatura Internacional em Ciência da Saúde (MEDLINE) e Scientific Electronic Library Online (SCIELO). Como também, livros que estavam disponíveis nos acervos bibliográficos, da biblioteca central do Centro Universitário de Ensino Superior de Maceió (CESMAC), foram utilizados.

Para tal estudo, foram utilizados determinados critérios importantes, a citar: a elaboração da pesquisa necessitaria de ensaios clínicos realizados de maneira controlada, randomizada, em pacientes que apresentavam algum problema de DTM. Foram selecionados estudos nos idiomas português, espanhol e inglês; fazendo uso de trabalhos expostos nos últimos 6 anos (2016 a 2021); que mencionavam a utilização da laserterapia nas clínicas odontológicas, apresentando como processo de exclusão os artigos do tipo relato de caso, séries de casos, ensaios que não apresentavam controle, artigos de revisão, monografias e os artigos científicos que não detinham importância com o tema estabelecido.

Foram eleitos os artigos que mostravam na sua integralidade o verdadeiro objetivo da pesquisa, atentandose para a relevância da importância do laser no tratamento de pacientes portadores de DTM, buscando, assim, observar as melhores condutas para a obtenção de seguimentos oportunos e vantajosos para os pacientes. Os descritores utilizados foram: Terapia a laser, Terapia com luz de baixa intensidade, Transtornos da Articulação Temporomandibular.

Os artigos foram escolhidos por 4 pesquisadores. No início, foram realizadas leituras sobre o conteúdo, e posteriormente, estabelecida uma leitura criteriosa dos resumos para, posteriormente, realizar a sondagem cautelosa das restrições. Logo depois, foi efetuada a leitura de todos os artigos, dos quais foram extraídos os subsídios de interesse, sendo analisadas as listas de referências, objetivando encontrar os artigos complementares para o engrandecimento científico da revisão. Com os artigos selecionados, foram tabuladas as diretrizes como o tamanho da amostragem, o verdadeiro objetivo, sua metodologia utilizada, os fatores analisados e os indispensáveis resultados.

A princípio, nessa respectiva revisão, foram estabelecidos 22 artigos que se demonstrava totalidade e completude nas especificas bases de dados. Dos quais, 10 estavam repetidos nas mencionadas fontes de pesquisa, ficando 12 artigos para o processo avaliativo do respectivo conteúdo, no entanto 3 foram excluídos por não apresentarem de maneira adequada as diretrizes fundamentais da construção do estudo. Em consequência 9 artigos foram incluídos para a interpretação e avaliação por intermédio das sínteses presentes, dos quais 3 foram excluídos, desta forma, foram definidos 6 artigos para o esclarecimento e constituição imprescindíveis para o progresso do estudo.

Depois que foram feitos os ensejos do respectivo estudo, ausentou-se outra fonte de pesquisa. $O$ fluxograma da Figura 1 menciona todas as formas de análises que foram produzidas mediante de apuramento feito por pesquisas determinadas para a validação direta desta análise literária.

Figura 1 - Fluxograma dos estudos identificados.

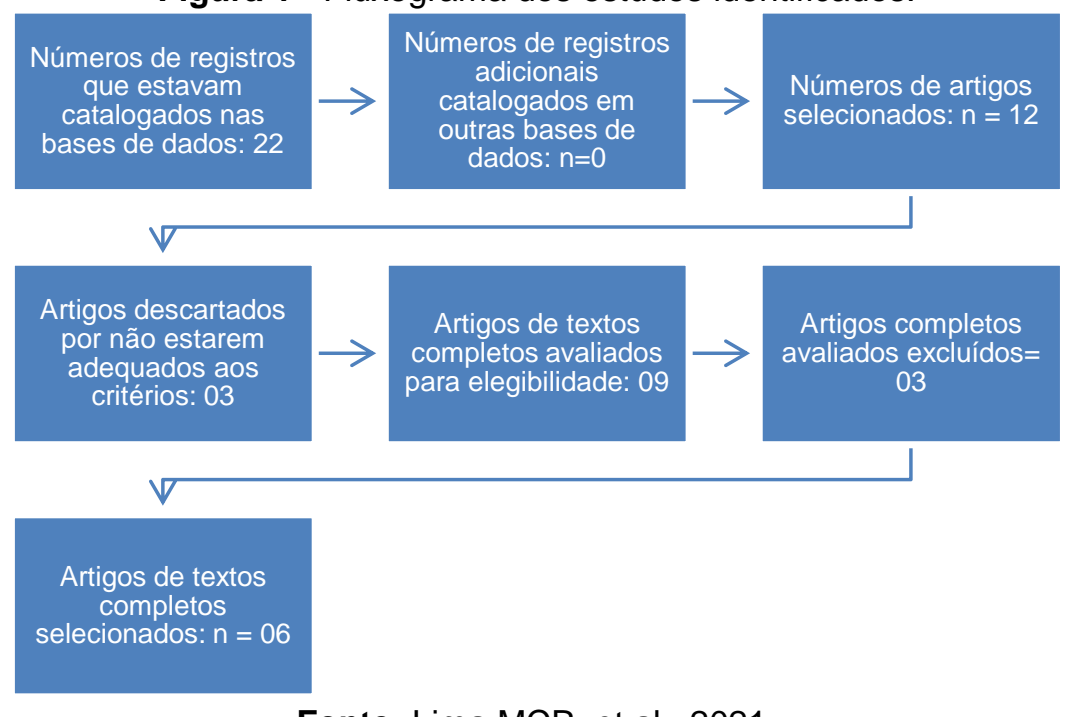

Fonte: Lima MCR, et al., 2021. 


\section{RESULTADOS E DISCUSSÃO}

\section{Limitação de abertura e travamento ao fechar ou abrir a boca}

É importante averiguar se a insuficiência de abertura da cavidade bucal é ocasionada por problemas de articulação ou musculatura. Durante o exame clínico, se ocorrer flexibilidade de maneira evolutiva, provavelmente a razão será as dores musculares. Já na ocorrência de inflexibilidade e resistência há maior aspecto de tratar-se de distúrbio articular, assim como a deslocação de disco sem possuir atenuação. Sabendo-se do quadro real do paciente, encaminha-o para fazer o tratamento e analisa-se o melhor procedimento a ser realizado, optando-se inúmeras vezes pela laserterapia (BRASIL, 2019; DE MEURECHY N e MOMMAERTS MY, 2018).

A Figura 2 demostra a avaliação do quadro clínico da DTM do paciente. É levado em consideração a realização de abordagem com terapias básicas, o acompanhamento periódico do paciente e o reforço de maneira positiva das condutas que devem ser feitas.

Figura 2 - Quadro clínico da DTM do paciente.

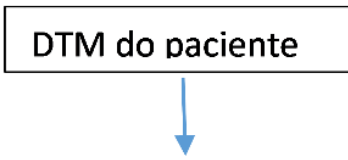

Fazer uma abordagem com terapias básicas: TCC (biofeedback, restrição de dieta, limitação de $A B$ ), exercícios básicos, automassagem, termoterapia, AINE/AIE e analgésicos.

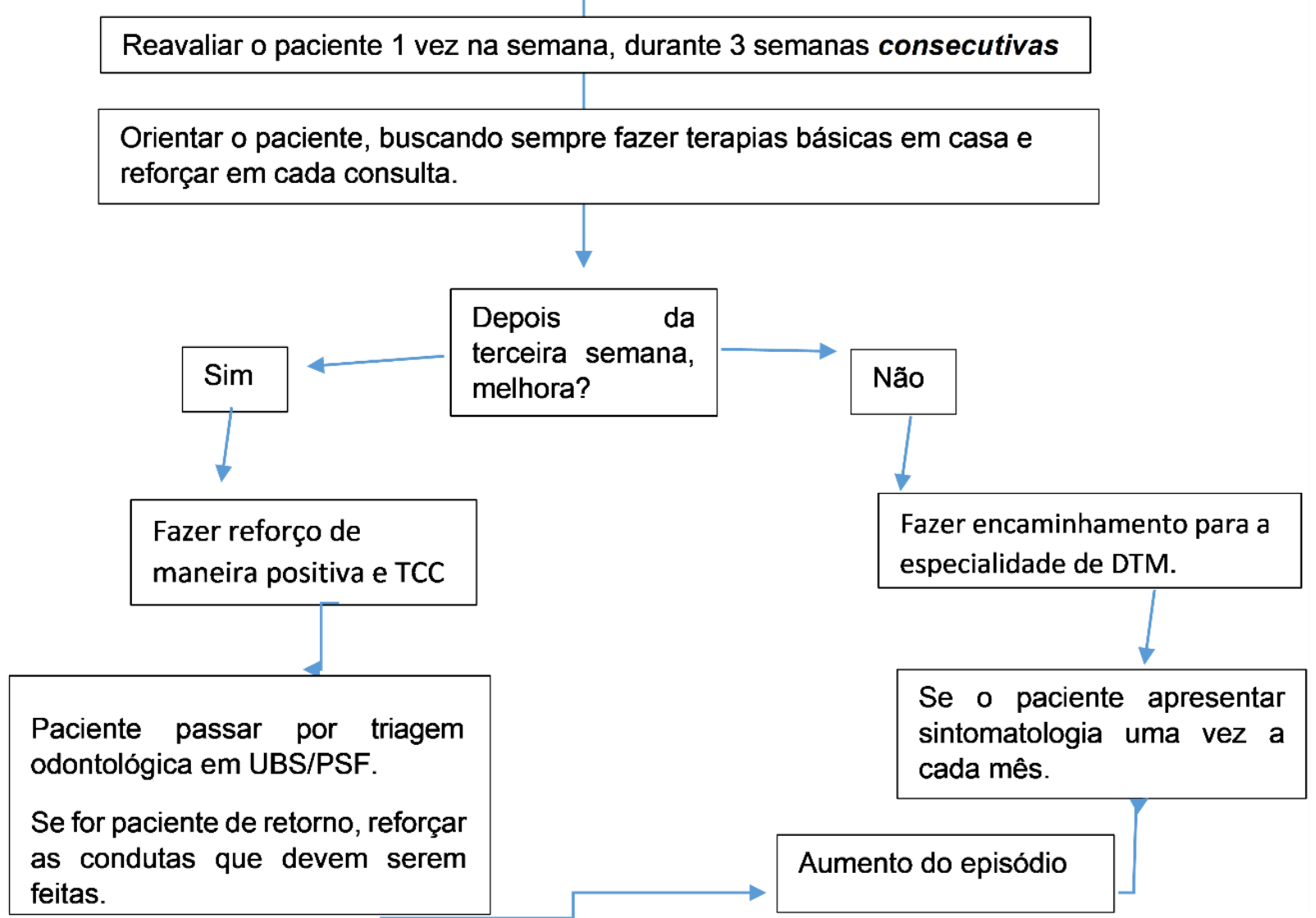

Fonte: Lima MCR, et al., 2021.

Os artigos possuíram suas informações através dos tópicos: autor/ano, país, objetivo e resultados, os quais foram tabulados e apresentados na Quadro 1. 
Quadro 1 - Delineamento, métodos e principais desfechos dos estudos selecionados.

\begin{tabular}{|c|c|c|c|}
\hline Autor/Ano & País & Objetivo & Resultados \\
\hline KHAW CM, et al., 2018. & Austrália & $\begin{array}{l}\text { Investigar o efeito da terapia a laser de baixa } \\
\text { intensidade (LLLT) no reparo da reabsorção } \\
\text { radicular inflamatória induzida ortodonticamente } \\
\text { (OIIRR). }\end{array}$ & $\begin{array}{l}\text { O volume total da cratera foi de } 0,746 \mathrm{~mm} 3 \text { para os dentes tratados com laser e } \\
0,779 \mathrm{~mm} 3 \text { para o sham. Houve uma diferença média de } 0,033 \pm 0,39 \mathrm{~mm} 3(95 \% \\
\text { Cl, }-0,21 \text { a } 0,148 \mathrm{~mm} 3 \text { ) maior volume da cratera de reabsorção no grupo sham em } \\
\text { comparação com o grupo laser; isso não foi estatisticamente significativo }(P= \\
\text { 0,705). Nenhum dano foi observado. }\end{array}$ \\
\hline CAVALCANTI MFXBC, et al., 2016. & Brasil & $\begin{array}{l}\text { Revisar sistematicamente os estudos que } \\
\text { investigaram o efeito da terapia a laser de baixa } \\
\text { intensidade (LLLT) nos níveis de dor em indivíduos } \\
\text { com DTM. }\end{array}$ & $\begin{array}{l}\text { Após uma revisão cuidadosa, } 14 \text { estudos se enquadraram nos critérios de inclusão, } \\
\text { dos quais } 12 \text { usaram um grupo placebo. Quanto ao protocolo de aplicação do laser, } \\
\text { a densidade de energia utilizada variou de } 0,9 \text { a } 105 \mathrm{~J} / \mathrm{cm} 2 \text {, enquanto a densidade } \\
\text { de potência variou de } 9,8 \text { a } 500 \mathrm{~mW} \text {. O número de sessões variou de } 1 \text { a } 20 \text { e a } \\
\text { frequência das aplicações variou de diária por } 10 \text { dias a } 1 \text { vez por semana por } 4 \\
\text { semanas. Uma redução nos níveis de dor foi relatada em } 13 \text { estudos, com } 9 \text { deles } \\
\text { ocorrendo apenas no grupo experimental e } 4 \text { estudos relatando o alívio da dor para } \\
\text { o grupo experimental e para o placebo. }\end{array}$ \\
\hline FARIAS RD, et al., 2016. & Brasil & $\begin{array}{l}\text { Avaliar o efeito do uso da terapia a laser de baixa } \\
\text { intensidade (LLLT) no controle da dor e } \\
\text { desconforto durante o tratamento ortodôntico. }\end{array}$ & $\begin{array}{l}\text { Uma diferença estatisticamente significativa foi observada }(P<0,005) \text { na redução } \\
\text { do desconforto no grupo exposto em comparação com o grupo placebo. Essa } \\
\text { redução do desconforto no GE foi observada em todos os intervalos de tempo. }\end{array}$ \\
\hline GODOY CHL, et al., 2017. & Brasil & $\begin{array}{l}\text { Avaliar a atividade muscular em indivíduos com } \\
\text { diagnóstico de DTM antes e após o tratamento com } \\
\text { laserterapia de baixa potência (LLLT) por meio do } \\
\text { uso da eletromiografia (EMG). }\end{array}$ & $\begin{array}{l}\text { Durante a avaliação isométrica dos músculos masseter e temporal anterior, foi } \\
\text { encontrado um aumento na média do sinal EMG no grupo submetido à LLLT ativa. } \\
\text { Quando avaliados individualmente, alguns participantes do grupo LLLT ativo } \\
\text { demonstraram uma redução na atividade muscular, mas não foram encontradas } \\
\text { diferenças significativas no sinal EMG médio entre as avaliações inicial e final. }\end{array}$ \\
\hline MELCHIOR MO, et al., 2017. & Brasil & $\begin{array}{l}\text { Analisar o efeito da associação da laserterapia de } \\
\text { baixa intensidade ao uso da placa oclusal como } \\
\text { tratamento para disfunção temporomandibular. }\end{array}$ & $\begin{array}{l}\text { Houve diminuição significativa da dor à palpação e da dor relatada de acordo com } \\
\text { a autopercepção dos sinais e sintomas para ambos os grupos tratados, porém de } \\
\text { forma mais acentuada para o GPL. Houve aumento da amplitude dos movimentos } \\
\text { mandibulares com diferença significativa após os tratamentos para ambos os } \\
\text { grupos. }\end{array}$ \\
\hline TORRES GM, et al., 2016. & Espanha & $\begin{array}{l}\text { O estudo pretendeu investigar os efeitos } \\
\text { terapêuticos da terapia a laser e de uma tala de } \\
\text { estabilização oclusal na redução da dor e } \\
\text { disfunção e na melhoria da qualidade do sono em } \\
\text { pacientes com DTM e SFM. }\end{array}$ & 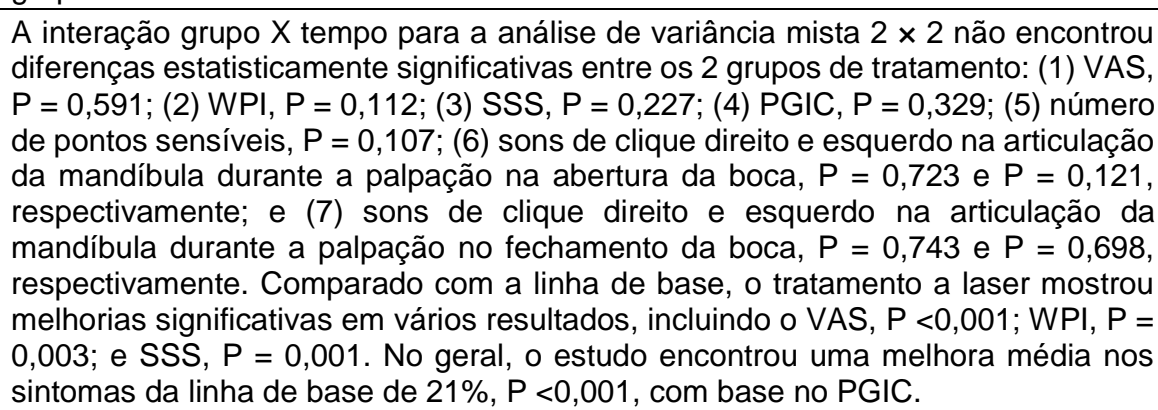 \\
\hline
\end{tabular}

Fonte: Lima MCR, et al., 2021.

REAS | Vol.13(4) | DOI: https://doi.org/10.25248/REAS.e7281.2021 Página $\mathbf{5}$ de $\mathbf{9}$




\section{A importância do laser na Odontologia}

A utilização da LBI vem sendo administrada para o tratamento de diversas patologias que precisam de bioestimulação, tendo como um dos principais critérios o alívio das dores agudas ou crônicas que os pacientes apresentam, sendo observado que depois de algumas sessões ocorre a diminuição de processos inflamatórios presentes no local estabelecido e a restauração da função. Esse tratamento terapêutico tem ofertado alto grau de segurança tanto para o paciente, quanto para o profissional, visto que não apresenta conduta invasiva, muito menos contraindicações, sendo também de fácil manuseio pelo profissional qualificado (COSTA DR, et al., 2017).

O laser de baixa intensidade exibe inúmeras características marcantes durante a utilização pelos Cirurgiões-Dentistas com fins preventivos e nas condutas relacionadas ao tratamento de transtornos orofaciais, como: processos relacionados a mucosites; que são inflamações da parte interna da boca e da garganta, podendo gerar úlceras dolorosas e feridas nessas regiões; aftas dolorosas, sensibilidades em tratamento restauradores, na DTM, nevralgias reconhecidas pela dor aguda e impactante que seguem o caminho de um nervo, pouca salivação, entre outros (FARIAS RD, et al., 2016).

A DTM determina-se como sendo uma terminologia coletiva da qual apresenta o envolvimento de um elevado número de discussões clínicas que sensibiliza os músculos responsáveis pela mastigação, a ATM e estruturas em que se encontram agregadas, ou os dois. Dos sinais mais presentes estabelecidos pelas disfunções da ATM, encontram-se sofrimento do paciente, em razão das dores presentes nos músculos da mastigação ou na ATM, alguns barulhos articulares, limitação na abertura da boca, retrações gengivais, maneira incorreta de oclusão, problemas relacionados à audição, dores de cabeça, sensibilidade em toda musculatura do sistema estomatognático (mandíbula, maxila, arcos dentários, ATMs e osso hióide) e cervicais (MAIOR BSS, et al., 2015).

O sistema mastigatório é constituído por diversos elementos, sendo observado que cada um, de maneira particularizada, exibe um suporte estrutural de maneira específica. Os músculos mastigatórios são compreendidos pelo masseter, temporal, pterigoideo lateral e medial. A ATM, os elementos dentais e os sistemas estruturais dos mesmos são conhecidos por apresentarem um sistema de menor flexibilidade. Dessa maneira, a susceptibilidade relacionada às dores nos sistemas de suportes supracitados aumenta, ocasionando uma limitação da mobilidade da mandíbula, por causa da grande quantidade de atividades musculares, gerando o bloqueio do fluxo sanguíneo habitual aos tecidos, ocasionando um acervo do sistema metabólico das células de tecidos musculares, provocando assim um estado de espasmo, cujas contrações involuntárias do músculo acarretam em fadiga e dores nos pacientes (DE MEURECHY N e MOMMAERTS MY, 2018).

\section{Laserterapia na DTM}

Por se tratar de uma doença que apresenta uma alta complexidade, a DTM tem gerado inúmeras variedades de recursos terapêuticos, tornando-se o mais efetivo, aquele proposto de maneira conjunta com uma equipe multiprofissional. Os agentes físicos frequentemente agregados para um bom tratamento são: termoterápicos, eletroterápicos, ultrassonografia, iontoforese, alguns fármacos que provocam analgesia e o laser. No entanto, a terapia com laser de baixa intensidade vem auxiliando nos tratamentos que apresentam sintomas dolorosos, possibilitando um nível de bem-estar admissível ao paciente, instantes depois da sua utilização. A LBI apresenta um tratamento decorrente através de uma radiação estabelecida na porção exposta do espectro presente, nas correntes de radiações eletromagnéticas, entre a luz infravermelha e o ultravioleta, e a dimensão da onda baseia-se no tipo de elemento estimulado (FERREIRA JB, et al., 2015; MAIOR BSS, et al., 2015).

A laserterapia é considerada uma categoria de tratamento da qual trata os pacientes, obtendo resultados positivos e não apresenta invasibilidade, além de ser de baixo custo. Nos últimos anos, vem tornando-se cada vez mais empregada nas condutas clínicas, tendo como objetivo aliviar a dor e atuar na reabilitação da regeneração dos tecidos. Dentre os resultados estabelecidos no tratamento, são utilizados: anti-inflamatórios, analgésicos, cicatrizantes e moduladores das atividades celulares, dos quais têm confiabilidade em inúmeras 
pesquisas cientificas. Em meio as condutas mais apropriadas, encontra-se o laser que emprega Arseneto de Gálio (AsGa) e Arseneto de Gálio e Alumínio (AsGaAl), que são compostos químicos sintéticos de materiais semicondutores, dos quais detêm benefícios nos tratamentos dos distúrbios musculoesqueléticos, por meio do fato de seu controle na forma de penetrar ser proporcionalmente maior, tendo seus princípios ativos força para atingir as estruturas musculares mais profundas (CATÃO MHCV, et al., 2012).

Os avanços dos estudos feitos na área da odontologia proporcionaram a utilização do laser com maior clareza e objetividade. Para isso, foram relacionadas inúmeras pesquisas através dos feixes de luz propagados, tendo maior ênfase o laser que detinha feixes de luz com menor intensidade, sendo observado que estes apresentavam resultados com melhor satisfação. Foi averiguado que a presença das luzes monocromáticas (aquelas que apresentam radiações eletromagnéticas na faixa de luz visível compostas por um único comprimento de onda) são satisfatoriamente aptas de conseguirem penetrar nas camadas dos tecidos com maior precisão e profundidade no tecido epitelial, causando assim a estimulação dos fotoceptores presentes, promovendo superiores estímulos não só das atividades funcionais, como também das células metabólicas do sistema célula, viabilizando seguimentos aceitáveis como é o caso dos efeitos analgésicos, anti-inflamatórios e de fontes bioestimulantes para diminuir as dores dos pacientes (COSTA DR, et al., 2017).

Antigamente, a compreensão de apenas a oclusão ser responsável por causar a DTM foi um dos marcos para os tratamentos aplicados de maneira mais invasiva e irreversível, como, por exemplo, grandes reajustes oclusais, mecanoterapias ortodônticas; que observa e trata as lesões neuro-músculo-esqueléticas; análise das alterações da postura e do equilíbrio mandibular, e até processos cirúrgicos para melhorar o agrupamento da ATM. Atualmente, com um melhor conhecimento sobre a DTM, sabendo-se que pode ser ocorrida por problemas multifatoriais, os profissionais optam por fazerem um tratamento reversível e não invasivo, através de uma boa anamnese nos pacientes; tendo bastante atenção nos sinais e sintomas de cada um, de maneira particularizada; utilizando uma abordagem multiprofissional e interdisciplinar. Nesse cenário, a LBI vem sendo utilizada como um meio físico no processo terapêutico da DTM, por causa dos seus resultados positivos (MATIAS AGC, et al., 2014).

A dimensão da onda, ou seja, a escolha do laser que irá ser utilizado precisa ajustar-se a necessidade de cada paciente. Assim sendo, o Arseneto de Gálio $(\mathrm{GaAs}=904 \mathrm{~nm})$ é uma das alternativas, porém deve ser utilizado para problemas ou doenças que apresentam maiores graus de profundidade, tais como, em edemas e dores no pós-operatório. Já o Arseneto de Galio e Alumínio (GaAlAs $=790 \mathrm{~nm}$ ou $830 \mathrm{~nm}$ ) é utilizado, geralmente, para inchaço, analgesia e regeneração da parte nervosa. A potencialidade trata-se da dosagem de fótons que atingem o local de atuação e, para ser calculada a quantidade correta do tempo e da densidade ou perspicácia de sua potencialidade, é fundamental ter o domínio sobre o conhecimento da potência média do laser que irá administrar. Se a conduta estabelecida for por motivos pulsados, a potência diversifica entre o grau máximo e valor mínimo, cabendo ao profissional saber o tempo correto, como também a média e sua continuidade (DE MEURECHY N e MOMMAERTS MY, 2018; MATOS AS, 2019).

O fundamental propósito do tratamento, tendo como base os sinais e sintomas dos pacientes, é de precaver as dores crônicas provocadas pela DTM, minimizando-as para, assim, restaurar a funcionalidade e elaborar um planejamento em que o paciente volte a executar suas atividades cotidianas com melhor bemestar (MELCHIOR MO, et al., 2017).

\section{Importância do laser na DTM}

O resultado analgésico provocado pelo tratamento com a LBI é a fundamental causa para sua utilização nas DTMs, levando-se em consideração que apresenta resultados mais rápidos do que a administração de medicamentos de origem farmacológica e até mesmo de placas oclusais (BROCHINI APZ, 2012). Cavalcanti MFXBC, et al. (2016), em suas pesquisas observaram que a atenuação das dores é estabelecida pela liberação de beta-endorfinas que é um neurotransmissor endógeno encontrado tanto nos neurônios do sistema nervoso central, quanto nos do sistema nervoso periférico.

Magri LV, et al. (2017), estabeleceram uma outra possibilidade, no que se refere aos efeitos de analgesia que o laser apresenta, ressaltando a existência do bloqueio da ciclo-oxigenase (COX) que é uma glicoproteína 
dimérica integral da membrana, encontrada predominantemente no retículo endoplasmático. Assim, atua como efetor secundário na via metabólica da cascata do ácido araquidônico, reduzindo dessa maneira as substâncias pró-inflamatórias encontradas na região específica.

Além da apresentação do efeito analgésico, eles observaram os benefícios da laserterapia nos relatos dos pacientes quando estes se referiam a uma diminuição parcial ou total dos sintomas dolorosos, gerando uma menor utilização de fármacos, com bons resultados em todas as faixas etárias, sendo um processo terapêutico indolor de forma não invasiva e custo recompensador, tanto para o profissional quanto para o paciente (MAGRI LV, et al., 2017).

Um estudo que deve ser levado em consideração é a verificação dos resultados quando utilizado produto placebo. Uma boa relação entre $\circ \mathrm{CD}$ e o paciente, juntamente com a apresentação de "alta tecnologia" agregada ao laser, traz um resultado placebo benéfico (BROCHINI APZ, 2012; TORRES GM, et al., 2016).

Ainda assim, é possível fazer interpelações sobre a explicação pela qual poucos pacientes não possuíram um resultado satisfatório ao tratamento. Em alguns acontecimentos, os pacientes que passaram pelo tratamento, demonstraram agravamento dos sintomas, sendo indispensável, dessa maneira, o reajuste no graduamento da dosagem ou até mesmo ser levado em consideração o intervalo entre uma sessão e outra para a utilização do laser. É possível considerar que algumas condições presentes na ATM não consigam corresponder de maneira satisfatória no mesmo padrão utilizado em outros pacientes. Daí a importância de estabelecer uma técnica particularizada para cada pessoa. Alguns fatores devem ser levados em consideração na hora do tratamento terapêutico feito com a LBI, a citar: estresse no cotidiano, a duração e tempo do avanço da patologia, como também perda grave relacionada à dimensão vertical que determinam de maneira negativa a eficiência analgésica da LBI (FERREIRA CLP, et al., 2016).

Godoy $\mathrm{CHL}$, et al. (2017), determinaram por meio de seus estudos que a maneira certa de se aplicar a laserterapia através da sua dosagem diária ou semanal é um dos fatores principais para que ocorra o resultado benéfico do tratamento. Provavelmente, seja esse o motivo pelo qual alguns pacientes dos estudos experimentais ainda estejam com um quadro sintomático mesmo depois de algumas sessões de utilização da LBI. É estabelecido, portanto, experimentos e estudos em que analisam de maneira significativa a efetividade de protocolos de administração controlados da LBI nos pacientes que apresentam dores tanto moderadas como severas.

Assim sendo, diante das análises dos estudos estabelecidos, a utilização do LBI demostrou-se como sendo uma ótima possibilidade nos procedimentos terapêuticos, principalmente quando refere-se as intervenções terapêuticas de desordens da região orofacial, incluindo principalmente na região da articulação temporomandibular. Esse recurso ajuda na diminuição das dores, em que promove bem-estar para os pacientes minutos após sua aplicação. A utilização no tratamento da DTM, tem como uma das principais vantagens ser um tratamento não invasivo e de pequeno custo. Além do que, sua utilização nas clinicas odontológicas, assegura a redução da procura de pacientes às cirurgias ou ao uso de fármacos para tratamento de dores e regeneração tecidual. A aplicação da LBI em indivíduos que apresentam DTM, vem proporcionalizando aos pacientes uma melhora significativa principalmente quando é levado em consideração a qualidade de vida (CATÃO MHCV, et al., 2012).

\section{CONSIDERAÇÕES FINAIS}

Ao ser instituída uma aprofundada revisão de literatura de caráter narrativo e descritivo dos artigos, objetivando estabelecer melhor particularidade científica do específico assunto, considerou-se que a implantação da laserterapia de baixa intensidade para o tratamento da disfunção temporomandibular é uma das terapias mais utilizadas pelos Cirurgiões-Dentistas em razão da resposta positiva nos tratamentos coadjuvantes de pacientes que apresentam quadros clínicos de DTM e da ausência de efeitos colaterais e de prejuízo as células sadias do indivíduo, tornando-se confiável. Porém, é vital a capacitação profissional para o manuseio do laser de baixa intensidade e a realização mais estudos randomizados, visando estabelecer um protocolo padronizado, respeitando a individualidade de cada paciente. 


\section{REFERÊNCIAS}

1. BRASIL. Manual de Dor Orofacial e Disfunção Temporomandibular para Cirurgiões Dentistas do SUS. 2019. Disponível em: https://www.prefeitura.sp.gov.br/cidade/secretarias/upload/saude/arquivos/11-04-VERSAO-FINALMANUAL-OROFACIAL.pdf . Acessado em: 20 de fevereiro de 2021.

2. BROCHINI APZ. Análise da contribuição do laser de baixa intensidade como terapia de apoio no tratamento de DTM com placa oclusal. Dissertação (Mestrado em Odontologia Restauradora) - Faculdade de Odontologia de Ribeirão Preto, Universidade de São Paulo, Ribeirão Preto, 2012: 179.

3. CATÃO MHCV, et al. Avaliação da eficácia do laser de baixa intensidade no tratamento das disfunções temporomandibulares: estudo clínico randomizado. Rev CEFA, 2012; 15(6): 1601-1608.

4. CAVALCANTI MFXBC, et al. Comparative study of the physiotherapeutic and drug protocol and low-level laser irradiation in the treatment of pain associated with temporomandibular dysfuction. Photomedicine and laser Surgery, 2016; 34(12); 652-656.

5. CIEPLIK F, et al. Antimicrobial photodynamic therapy - what we know and what we don't.Critical Reviews in Microbiology, 2018; 44(5): 571-589.

6. COSTA DR, et al. Efeito da terapia LED na disfunção temporomandibular: estudo de caso. Ver Scientia Médica, 2017; 27(2); 37-42.

7. DE MEURECHY N, MOMMAERTS MY. Alloplastic temporomandibular joint replacement systems: a systematic review of their history. Int J Oral Maxillofac Surg., 2018; 47(6):743-754.

8. FARIAS RD, et al. Evaluation of the use of low-level laser therapy in pain control in orthodontic patients: A randomized split-mouth clinical trial. Angle Orthodontist, 2016; 86(2): 193-198.

9. FERREIRA CLP, et al. Sinais e sintomas de desordem temporomandibular em mulheres e homens. CoDAS, 2016; 28(1): 17-21.

10. FERREIRA JB, et al. Tratamento fisioterapêutico nas disfunções temporomandibulares. Rev InterScientia. 2015; 3(1): 123-146.

11. GODOY CHL, et al. Electromyographic evaluation of a low-level laser protocol for the treatment of temporomandibular disorder: a randomized, controlled, blind trial. J. Phys Ther Sci., 2017; 29(12): 2107-2111.

12. KHAW CM, et al. Physical properties of root cementum: Part 27. Effect of low-level laser therapy on the repair of orthodontically induced inflammatory root resorption: A double-blind, split-mouth, randomized controlled clinical trial. American Journal of Orthodontics and Dentofacial Orthopedics, 2018; 3(154): 326 -336.

13. MAGRI LV, et al. Effectiveness of low-level therapy on pain intensy, pressure pain threshold, and SF-MPQ indexes of women with myofascial pain. Ver Lasers Med Sci., 2017.

14. MAIOR BSS, et al. Laserterapia de Baixa Intensidade no Tratamento de Laserterapia de Baixa Intensidade no Tratamento de Desordens Temporomandibulares Desordens Temporomandibulares, Fac. Odontol. Porto Alegre, Porto Alegre, 2015; 48, (1/3): 88-91.

15. MATIAS AGC, et al. Modulação da dor em portadores de disfunções temporo-mandibulares pela ação do laser AsGaAl. Rev Inter Scientia, 2014; 2(2): 25-37.

16. MATOS AS. Laserterapia no controle da disfunção temporomandibular dolorosa: evidências científicas. Dissertação apresentada à faculdade de Odontologia de Bauru, São Paulo, 2019.

17. MELCHIOR MO, et al. Laserterapia de baixa intensidade associado ao uso de placa oclusal no tratamento de disfunção temporomandibular: estudo clínico randomizado. Rev Dor, 2017; 18(1): $12-7$.

18. POL R, et al. Efficacy of Anti-Inflammatory and Analgesic of Superpulsed Low Level Laser Therapy After Impacted Mandibular Third Molars Extractions. Journal of Craniofacial Surgery, 2016; 27(3): 685-690.

19. SANT'ANNA EF, et al. High-intensity laser application in Orthodontics. Dental Press Journal of Orthodontics, 2017; 22(6): 99-109.

20. TORRES GM, et al. Laser therapy and occlusal stabilization splint for temporomandibulardisorders in patients with fibromyalgia syndrome: A randomized, Clinical trial. Alternative therapies heath Med., 2016; 22(5): 23-31. 